\title{
The Extinction Toward the Galactic Bulge from RR Lyrae Stars
}

\author{
Andrea Kunder \\ E-mail: andrea.m.kunder@dartmouth.edu \\ Dartmouth College, 6127 Wilder Lab, Hanover, NH 03755 \\ Piotr Popowski \\ E-mail: popowski@mpa-garching.mpg.de \\ Max-Planck-Institut für Astrophysik, Karl-Schwarzschild-Str. 1, Postfach 1317, 85741 \\ Garching bei München, Germany \\ E-mail: Andrea.M.Kunder@Dartmouth.edu and popowski@mpa-garching.mpg.de \\ Kem H. Cook \\ IGPP/LLNL, P.O.Box 808, Livermore, CA 94551, USA \\ E-mail:kcook@igpp.ucllnl.org \\ Brian Chaboyer \\ Dartmouth College, 6127 Wilder Lab, Hanover, NH 03755 \\ E-mail: chaboyer@heather.dartmouth.edu
}

\begin{abstract}
We present mean reddenings toward 3525 RR0 Lyrae stars from the Galactic bulge fields of the MACHO Survey. These reddenings are determined using the color at minimum $V$-band light of the RR0 Lyrae stars themselves and are found to be in general agreement with extinction estimates at the same location obtained from other methods. Using 3256 stars located in the Galactic Bulge, we derive the selective extinction coefficient $R_{V, V R}=A_{V} / E(V-R)=4.3 \pm 0.2$. This value is what is expected for a standard extinction law with $R_{V, B V}=3.1 \pm 0.3$.
\end{abstract}

Subject headings: dust, extinction — Galaxy: center — stars: statistics — surveys 


\section{Introduction}

Studies of stars in the central Galactic region are essential for an understanding of the Milky Way. For example, the questions of the structure and extent of the Galactic bar, the star formation history, and the distance to the center of our Galaxy can all be answered by analyzing various stellar tracers. Knowledge of the Galactic extinction is mandatory to begin investigations of these stars in a quantitative fashion. A good understanding of extinction is particularly important for the direction toward the Galactic Bulge and within the Galactic plane, where extinction is very significant.

Extinction $A_{\lambda}$ changes as a function of wavelength $\lambda$. The shape of the extinction curve is characterized by the quantity $R_{V}$, the ratio of total to selective extinction; Cardelli et al. (1989) have shown that the family of curves characterized by this single parameter provides a good fitting approximation to most extinction curves. Thus, if the value of $R_{V}$ can be determined (e.g., from optical and IR photometry), then one can approximately find the properties of the entire UV-IR extinction curve.

There are a few suggestions that the coefficient of selective extinction in the direction of the Galactic Bulge may be non-standard (e.g. Popowski 2000). Anomalous extinction, i.e., extinction described by smaller $R_{V, B V}$ or $R_{V, V R}$ ratio than the standard value of $R_{V, B V}=$ 3.1, can significantly affect analysis of the stellar populations in the Galactic Bulge. Here, $R_{V, B V}$ is the coefficient of selective extinction $A_{V} / E(B-V)$, with reference to the $B$ and $V$ filters, and $R_{V, V R}=A_{V} / E(V-R)$, alluding to the $V$ and $R$ filters. Especially with the onset of a new generation of large telescopes, this problem becomes increasingly pressing and important, and one that can be addressed with this study. Here, optical $V$ and $R$-band data for 3256 RR0 Lyrae star 1 from the MACHO microlensing experiment are used to find both the coefficient of selective extinction and to compute the absolute reddening near the Galactic bulge/bar. The value of $R_{V, B V}$ is known to vary in certain directions, and reddening laws are normally derived from differential measurements of reddened and unreddened luminous early-type stars. However, as such stars are rare in the Galactic Bulge, the normal derivation of $R_{V, B V}$ can not be carried out. The $R_{V, B V}$ values determined from the RR Lyrae stars are compared with other reddening relations.

The data used in this analysis are based on the MACHO photometry of 3674 RR0 Lyrae stars toward the Galactic Bulge. Cook, Kunder, \& Popowski (2007, in preparation). Cook et al. (2007, in preparation) divide the MACHO bulge RR0 Lyrae variables into those

\footnotetext{
${ }^{1} \mathrm{RR} 0$ stars have been traditionally called RRab stars and are simply fundamental pulsators. For the new, intuitive nomenclature, see Alcock et al. (2000).
} 
associated with the Galactic Bulge (3256 stars), and the ones associated with the neighboring Sagittarius dwarf galaxy (409 stars). Their classification is assumed in this work.

The structure of this paper is the following. The method and resultant coefficient of selective extinction toward the Bulge is discussed and compared to other estimates in $\S 2$. Reddenings of the Bulge RR0 Lyrae stars are determined in $\S 3$ using their minimum Vband light colors These reddenings are then compared to Popowski et al. (2003) reddening estimates and the outliers are discussed.

\section{Coefficient of Selective Extinction}

\subsection{General approach}

As the bulge RR Lyrae stars span a much larger range in color than Sgr ones, a coefficient of selective extinction is determined using only this subsample. Assuming that the extinction affects the $V$ magnitudes much more significantly than the distance spread, the intrinsic scatter in absolute magnitudes, and the scatter due to various observational errors, the slope of the stellar locus on a $V_{R R}$ (mean $\mathrm{V}$ magnitudes of the $\mathrm{RR}$ Lyrae stars) versus $(V-R)_{R R}$ (mean color of the RR Lyrae stars) color-magnitude diagram (CMD) yields $R_{V, V R} \equiv A_{V} / E(V-R)$ (Kunder et al. 2006). In other words, if in the distance modulus,

$$
V_{R R}-M_{V}-A_{V}=5 \log (d)
$$

we assume $M_{V}$ and $d$ is approximately constant, then the apparent magnitude, $V_{R R}$, is proportional to $A_{V}$ :

$$
V_{R R} \sim A_{V} \sim \frac{E(V-R)}{R_{V, V R}} .
$$

Taking the $(V-R)_{0}$ of each RR0 Lyrae star to be approximately constant,

$$
V_{R R} \sim \frac{(V-R)_{R R}}{R_{V, V R}}
$$

which is in the form of a general linear equation with $V_{R R}$ as the independent variable, $(V-R)_{R R}$ as the dependent variable, and $R_{V, V R}$ as the slope. It is shown in the following section that the above conditions are approximately met.

Figure 1 is a plot of mean $V_{R R}$, versus mean $(V-R)_{R R}$. Artificial star tests from Alcock et al. (1999) indicate that the limiting magnitude of the photometry in the $V$ and $R$ band is about 21.5, which is 0.5 magnitudes fainter than the faintest RR Lyrae star in this MACHO Bulge sample. The Monte Carlo analysis discussed later in this section indicates the limiting magnitude has little effect on this paper's analysis. 
The sources contributing to the intrinsic dispersions of the average $V$ and $R$ apparent magnitudes are now discussed and quantified following the approach from Clementini et al. (2003) .

1. photometric errors. Since many MACHO fields partly overlap on the sky, some stars may be counted in the database twice. Using 184 double-represented stars, the internal photometric uncertainty in $(V-R), V$, and $R$ was found by comparing colors and magnitudes evaluated in different fields. For the MACHO data toward the Galactic Bulge, the individual error in $V_{R R}$ is $\pm 0.15 \mathrm{mag}$ and the individual error in $(V-R)_{R R}$ is 0.04 (Cook et al. 2008, in preparation). 2 As the error in $V_{R R}$ and $(V-R)_{R R}$ are correlated, upon multiplying the error in $(V-R)_{R R},(0.04$ mags $)$, by the slope of the fit, (4.28), this value is added to the error in $V_{R R}$. The photometric error in the points in Figure 1 is thus 0.32 mags.

2. absolute magnitude of RR Lyrae stars. It is generally assumed that the absolute magnitude of an RR Lyrae star has a linear dependence on $[F e / H]$, and that $\Delta M_{V}(R R)$ / $\Delta[F e / H]=0.2 \mathrm{mag} \mathrm{dex}^{-1}$. The dispersion around the mean value of $[\mathrm{Fe} / \mathrm{H}]=-1.00$ dex found in Galactic Bulge RR Lyrae stars is $\sigma_{V}=0.29 \mathrm{mag}$. (Kunder et al. 2007, in preparation). This corresponds to an absolute magnitude dispersion of about 0.06 mag.

The level of evolution off the zero-age horizontal branch (ZAHB) can also affect at RR Lyrae star's absolute magnitude. From the vertical height of the HB of a number of globular clusters of different metallicities, the dispersion around the average RR Lyrae luminosity due to the evolution off the ZAHB of each individual RR Lyrae is estimated as $\sim 0.08 \mathrm{mag}$ (Clementini et al. 2003; Sandage 1990).

The scatter in RR Lyrae absolute magnitude due to evolutionary phase, helium content, and alpha-element abundance $[\alpha / \mathrm{Fe}]$, are less significant and are not accounted for.

3. bulge distance spread.

Using the standard Hernquist model density profile with a scale length, characteristic density, and characteristic velocity of the bulge from Widrow \& Dubinski (2005) Table 2, the size of the bulge is $\sim 1 \mathrm{kpc}$. As the galactic latitude and longitude of the MACHO bulge fields ranges from $-1.5^{\circ}$ to $-10^{\circ}$ and $0^{\circ}$ to $10^{\circ}$, respectively, a correction between the line of sight of the RR Lyrae stars in the bulge fields and the plane of the galaxy is negligible. A distance spread of $1 \mathrm{kpc}$ corresponds to a $\Delta V$ of $\sim 0.29$ mags.

Adding in quadrature all dispersion contributions, the scatter in $V$ for a fixed $(V-R)$

${ }^{2}$ For more information on the calibration of the MACHO Photometry RR Lyrae stars Database, see Alcock et al. (1999), especially pgs. 1547 and 1551. 
is 0.44 mags, with the major source of this scatter resulting from the extent of the bulge along the line of sight.

Fitting a straight line to the data must be done with caution, since errors are present in both independent and dependent variables and are furthermore correlated with each other. Ordinary least squares (OLS), which assumes no error in the independent variable, can therefore not be used here. Instead a straight line fit to the data is obtained using an OLS bisector fit to the individual data points.

The OLS-bisector method has been used when both variables are subject to measurement error and it is not clear which variable should be treated as the independent and which as the dependent. It has been shown to outperform other approaches in such cases (Babu \& Feigelson 1992; Isobe et al. 1990). Using the SLOPES program (Feigelson \& Babu 1992), the data are fit using the OLS-bisector method. The slope found is $4.28 \pm 0.04$ and is shown as a solid line in Figure1. Upon restricting the fit to those stars with $(V-R)>0.34$, a slope of $4.34 \pm 0.04$ is obtained. A further cut to remove the very red stars with $(V-R)>1.1$, yields a slope of $4.49 \pm 0.05$. These fit values are given in Table 1 , the $R_{V, V R}$ chosen as the optimal regression is $R_{V, V R}=4.28$, as it accounts for all data values. Table 1 shows that the slope is quite sensitive to just a few points, although these formal errors for the OLS-bisector method are quite small. The error in $R_{V, V R}$ of \pm 0.2 is chosen to encompass the range of slopes.

The OLS-bisector fit to our data is further investigated with a simple Monte Carlo analysis. To duplicate the observed RR0 Lyrae data in this paper, $3500 E(V-R)$ values between 0 and 1.5 are randomly selected. $E(V-R)$ is converted to Av using a selective extinction coefficient, $R_{V, V R}$, which we vary. Assuming an RR Lyrae absolute magnitude of $M_{V}=0.6$ and a distance to the Bulge of $8 \mathrm{kpc}$, an observed $V$ magnitude is found. As the observed $V$ magnitudes in the MACHO data have an error of $\sim 0.5$ mags, Gaussian random numbers are generated with a dispersion of 0.5 mags, and added to the simulated $V$ magnitudes. Using $(V-R)=E(V-R)+0.28$, and assuming the uncertainty in the measured $(V-R)$ colors is 0.05 magnitudes, the simulated $\mathrm{V}$ magnitudes can be plotted as a function of simulated (V-R) magnitudes. We make sure this simulated plot looks similar to Figure 1 .

Next a cut is made to remove all stars which have $\mathrm{V}$ and $\mathrm{R}$ magnitudes less than 21.5. The OLS-bisector method is then used to derive the slope of the fit before and after the magnitude cut. We find there is no significant change in the value of the slope after the cut. However, the slope found using the OLS-bisector method is not always the value of the slope that was input. This has implications on the derived $R_{V, V R}$ value. Apparently the errors in the photometry in this paper are sufficient to throw off the OLS-bisector fit for a given 
value.

Figure 2 shows the difference in the true $R_{V, V R}$ value and the OLS-bisector slope, $R_{(V, V R)}$-OLS slope, as a function of the true $R_{V, V R}$ value. The scatter in this plot is mainly due to the errors in the $V$-magnitudes and $(V-R)$ colors. A slope with a value of $\sim 4.6$ exhibits the least amount of deviation between the true and calculated $R_{V, V R}$ value. The $R_{V, V R}$ value found in this paper is close to 4.6, so the slope found by the OLS-bisector method should not be significantly biased. Even values well above and below 4.6 do not differ from the true $R_{V, V R}$ value by more than 0.2 mags. Other simulations with somewhat different input parameters (e.g., uncertainty in photometry) are performed and results similar, but not identical to Figure 2, are found. Thus, we can not simply correct the slope that is obtained from the real data using these simulations. Thus, an uncertainty of 0.2 in the $R_{V, V R}$ is adopted.

\subsection{Comparisons and Consistency}

It is an interesting question how this $R_{V, V R}$ value from the MACHO RR Lyrae stars compares with other reddening relations. Reddening relations determined from broad band photometric data depend on the intrinsic spectral energy distributions of the objects used to determine them as well as the particular filters. The MACHO $b_{M}$ and $r_{M}$ filters are considerably different from Johnson $V$ and Kron-Cousins $R$.

To compare our results to the standard extinction curve, the Fitzpatrick (1999) extinction curve with a constant total-to-selective extinction parameter, $R_{V B V}=3.1$, is used with the PHOENIX model synthetic spectra (Hauschildt, Allard \& Baron 1999). The Johnson BVR transmission curves from Bessell (1990) yield $R_{V, V R}=4.88$ for $T_{\text {eff }}=6000 \mathrm{~K}$, $R_{V, V R}=4.92$ for $T_{e f f}=7000 \mathrm{~K}$ and $R_{V, V R}=5.07$ for $T_{e f f}=30000 \mathrm{~K}$. An effective temperature of $6000 \mathrm{~K}-7000 \mathrm{~K}$ is typical of RR Lyrae stars, and an effective temperature of 30000 $\mathrm{K}$ is typical of early type stars. The synthetic spectra adopted is one with $[\mathrm{Fe} / \mathrm{H}]=-1.0$ and $\log g=2.5$, but varying $[\mathrm{Fe} / \mathrm{H}]$ and surface gravity does not significantly affect the $R_{V, V R}$ values.

The MACHO $b_{M}$ and $r_{M}$ filters are non-standard, but were transformed into the Johnson $V$ and Kron-Cousins $R$ as described in Alcock et al. (1999). We simulated this procedure by using the MACHO $b_{M}$ and $r_{M}$ transmission curves and our synthetic spectra to obtain MACHO $b_{M}$ and $r_{M}$ magnitudes. These were then converted using the Alcock et al. (1999) transformation. It is found that $R_{V, V R}=4.34$ for $T_{\text {eff }}=6000 \mathrm{~K}$, and $R_{V, V R}=4.26$ for $T_{\text {eff }}=7000 \mathrm{~K}$. Both these values are in agreement with the value obtained in this analysis. 
In comparison, a $R_{V, B V}=2.5$ would yield $R_{V, V R}=3.77$.

Reddening laws are normally derived from differential measurement of reddened and unreddened luminous early-type stars. But such stars are absent in the Galactic Bulge. This analysis uses Population II type stars and indicates that the reddening law in the bulge is on average similar to the standard solar neighborhood value of $R_{V, B V}=3.1$. Changing $R_{V, B V}$ to e.g. a non-standard value, does significantly affect the measured $R_{V, V R}$. Quantitatively, it is found that the change in $R_{V, V R}$ is $68 \%$ of the change in $R_{V, B V}$.

Using Galactic bulge Red Clump Giants from the OGLE dataset, Udalski (2003) present substantial evidence that the ratio of total to selective absorption, $R_{V, V I}$, is much smaller toward the Galactic Bulge than the value corresponding to the standard extinction curve and that $R_{V, B V}$ varies considerably along different lines of sight. Their value of $R_{V, V I} \sim$ 2.1, as opposed to the standard value of about 2.5, corresponds to an $R_{V, B V}$ of about 2.6. Stanek (1996) constructed extinction and reddening maps for Baade's window from colormagnitude diagrams obtained by OGLE which showed that extinction in Baade's window is quite irregular, varying between 1.3 and $2.8 \mathrm{mag}$ in $A_{V, B V}$, with an estimated error of $0.1 \mathrm{mag}$. Ruffle et al. (2004) use 70 Galactic planetary nebulae observed using narrow-band filters to find observed $\left\langle R_{V, B V}\right\rangle=2.0$ toward the bulge.

The $R_{V, V R}$ value determined from the RR Lyrae stars above, indicates that the standard Galactic reddening law can in general be adopted in studies of objects toward the bulge. We caution that the value of $R_{V, B V}$ can vary in certain directions but that on average, the standard reddening law can be used.

\section{Reddening}

\subsection{Determination of intrinsic $(V-R)_{0}$ colors}

Sturch (1966) and Blanco (1992) argue that apparent $(B-V)$ colors of RR Lyrae stars at phases close to the minimum light can be utilized to measure the amount of interstellar reddening along the line of sight to the star since the intrinsic $(B-V)_{0}$ colors seem constant in the phase interval $0.5-0.8$. Mateo et al. (1995) suggest that $(V-I)$ colors might also be used, perhaps with a smaller or negligible metallicity correction. We investigate this further for $(V-R)$ colors below.

The colors at minimum light, $(V-R)_{0}$, can be computed via two different methods. In the first method, the Fourier fits to both $V$ and $R$-band data are used to average the colors, in magnitude units, for phases between 0.5 and 0.8 . However, we find that when $(V-R)_{0}$ 
is determined using this method, it correlated with amplitude. In the second method, the $(V-R)$ color is found at minimum $V$-band light. Kanbur \& Fernando (2004) used this technique and found that intrinsic color at minimum $V$-band light is nearly independent of period and $V$-band variation amplitude. Since this method yields $(V-R)_{0}$ values that are very weakly correlated with amplitude, this is the method employed here. Table 2 presents our determination of unreddened $(V-R)_{0}$ colors for eleven well observed field RR0 variables (the data in Table 2 come from Liu \& Janes 1989; Cacciari et al. 1987; and Layden 2005 , private communication). The color excess, $E(V-R)$, is computed by multiplying the published $E(B-V)$ values by the ratio of $R_{V, B V} / R_{V, V R}=3.315 / 5.16$, where the values $R_{V, B V}=3.315$ and $R_{V, V R}=5.16$ are taken from Schlegel et al. (1998). 3 A plot of $E(V-R)$ vs $(V-R)$ has a linear character. This is quite remarkable, considering some of these stars exhibit the Blazhko effect (e.g., AW And), some are affected by both the Blazhko effect and a "phase-lag" problem (e.g., SS For), and some have evidence of shock waves in their atmospheres (e.g., RV Phe, V440 Sgr) as discussed by Cacciari et al. (1989a), Cacciari et al. $(1989 \mathrm{~b})$. It has been shown that in many RR Lyrae stars the Blazhko effect does not affect colors at minimum light, making this method robust. We conclude that the mean $(V-R)_{0}$ color of RR0 Lyrae stars at minimum $V$-band light is $0.28 \pm 0.02$. Table 2 suggests that the intrinsic color at minimum light is independent or at least very insensitive to metallicity.

\subsection{Reddening toward the bulge RR Lyrae stars}

The observed and intrinsic colors at minimum $V$-band are put forth to determine the color excess of the bulge RR0 Lyrae stars:

$$
E(V-R)=(V-R)-(V-R)_{0}
$$

The Fourier fits performed to find $(V-R)$ color at minimum $V$-band light are sensitive to the quality of a lightcurve. To insure high $E(V-R)$ accuracy without sacrificing the sample size, the Fourier fits in both passbands for all stars are individually examined. Only those with fits that approximated the RR Lyrae light curve nicely are taken (i.e., if the Fourier method mimicked features in the RR Lyrae lightcurve well, such as the dip and sharp rise of the curve at minimum light). This criterion results in 3525 RR0 Lyrae stars with well determined reddening values. Example entries of this reddening catalog are presented in Table 3 ; the complete listing is available in the electronic version.

\footnotetext{
${ }^{3}$ We explicitly assume here that for this sample of RR Lyrae stars, extinction is on average well represented by the standard $R_{V, B V}$ value.
} 
Multiplying the $3525 E(V-R)_{R R}$ values by the selective extinction coefficient, $R_{V, V R}$, allows the determination of the visual extinction. A map of the visual extinction, $A_{V}$, is shown in Figure 3, As Popowski et al. (2003) also remark, it is immediately obvious that on large scales, extinction is regularly stratified parallel to the Galactic plane.

To determine the distance modulus to the Galactic Bulge from the RR0 Lyrae stars, an RR Lyrae absolute magnitude of $\mathrm{M}_{\mathrm{V}}=0.59 \pm 0.03$ at $[\mathrm{Fe} / \mathrm{H}]=-1.5$ as compiled by Cacciari \& Clementini $(2003)$ is adopted. If a metallicity dependence of $\mathrm{M}_{\mathrm{V}}=(0.25 \pm$ $0.05)[\mathrm{Fe} / \mathrm{H}]+$ constant is adopted (consistent with the range of slopes found in the literature), then using the average metallicity of $[\mathrm{Fe} / \mathrm{H}]=-1$ from above, an $\mathrm{M}_{\mathrm{V}}=0.72 \pm 0.04$ for $\mathrm{RR}$ Lyrae stars in the Bulge is obtained. This predicts $(\mathrm{m}-\mathrm{M})_{0}=14.7 \pm 0.1$ for Bulge RR Lyrae. Adopting the RR Lyrae statistical parallax calibration of $\mathrm{M}_{\mathrm{V}}=0.77 \pm 0.13$ at $[\mathrm{Fe} / \mathrm{H}]=-1.6$ from Gould \& Popowski (1998), then using the same metallicity dependence, an $(\mathrm{m}-\mathrm{M})_{0}=14.5 \pm 0.16$ for RR Lyrae stars in the Galactic Bulge is predicted. This is identical to the Galactocentric distance modulus measured by Eisenhauer et al. (2003) from the orbit of a star around the central black hole.

\subsection{Comparison with complementary MACHO determination}

In this subsection the reddenings obtained here are tested against Popowski et al. (2003) values, which are based on photometry from the MACHO bulge fields. The MACHO data of the Galactic Bulge are based on observations of 94 bulge fields, each field covering an area of 43' by 43'. Popowski et al. (2003) use 4' x 4' sky regions (tiles) as resolution elements. They show that mean colors for these tiles can be converted to extinction, and can thus be used to derive a visual extinction map. Each RR0 Lyrae star is matched with the corresponding tile, and the color excess, $E(V-R)_{\mathrm{RR}}$, of the line of sight to the RR0 Lyrae star is compared to the Popowski et al. (2003), $E(V-R)_{\mathrm{CMD}}$, as calibrated to Stanek (1996) extinction 4 . Figure 4 shows this linear relationship, with the line of slope unity over-plotted. The $E(V-$ $R)_{\mathrm{RR}}$ values become larger than $E(V-R)_{\mathrm{CMD}}$ when reddening increases, which could mean the Popowski et al. (2003) extinction map underestimates the extinction in areas with high reddening. Performing the Bessell \& Germany (1999) calibration to the MACHO RR Lyrae lightcurve data does not alter this result. An increase in scatter is expected in stars with high reddenings, since generally a redder RR0 Lyrae star is fainter and is more likely to have noisy photometry and lightcurves. Especially near the Galactic center, differential reddening due

\footnotetext{
${ }^{4}$ The Popowski et al. (2003), $E(V-R)_{\mathrm{CMD}}$, as calibrated to Dutra et al. (2003) extinction is about 0.07 redder than $E(V-R)_{\mathrm{CMD}}$ calibrated to Stanek (1996).
} 
to nonuniform distribution of the intervening dust on small spatial scales makes the study of the stellar population difficult even in the infrared (see, e.g., Narayanan et al. 1996; Davidge 1998). Frogel et al. (1999) show in their Figure 5, that the amount of differential reddening in a field is directly proportional to the average reddening: the more reddening present in a field, the more patchy that reddening is. As the Popowski et al. (2003) extinction map is based on the average color of 4' x 4' sky regions, their average colors and hence reddenings would be susceptible to differential reddening, explaining the slight discrepancy between their reddenings and ours at higher reddenings.

What could cause the systematic bias in the $E(V-R)_{\mathrm{RR}}$ values to become larger than the $E(V-R)_{\mathrm{CMD}}$ as the reddening increases? Popowski et al. (2003) perform a calibration to Stanek (1996) extinction and this calibration assumes a uniform $R_{V, B V}$ value. This assumption does not hold, however, since the extinction toward the Bulge has nonstandard properties (Popowski 2000). Udalski (2003) find that the difference between the measured line of sight $R_{V, V I}$, and the standard value of $R_{V, V I}$ depends on color. Furthermore, they find that this difference is larger for redder $V-I$ colors and practically negligible in the range where the OGLE data were calibrated by standards $(V-I<2)$. Similarly, the difference in the coefficient of selective extinction $R_{V, V R}$ and the standard $R_{V, V R}$ would also increase with increasing $V-R$ and hence with increasing $E(V-R)_{C M D}$ (since Popowski et al. (2003) uses the average color to determine $\left.E(V-R)_{C M D}\right)$. As the Popowski et al. (2003) $E(V-R)_{C M D}$ calibration neglects a change in $R_{V, V R}$ with increasing color, at higher $E(V-R)_{C M D}$ the values will be underestimated.

\subsubsection{Outliers}

The most obvious outlier in Figure 4 is the RR0 Lyrae star that is designated in the MACHO database as 175.30921.95. It has a negative $E(V-R)_{R R}$, which is unphysical. It has a period, amplitude and light curve shape that confirm it is a fundamental mode pulsator. However, as is clear from its position on a color-magnitude diagram, with $(V-R)=0.21$ it is one of the bluest stars in the dataset (see Figure 11). Sturch (1966) suggests that as many as $3 \%$ of the RR0 Lyrae stars may have an unusual blueness associated with them. The only stars with a color bluer than this star and with an R-band lightcurve suitable for reddening determination, are stars 125.23192 .175 and 117.26079.4609, with $(V-R)=0.14$ and $(V-R)=0.19$, respectively. The bluer of these two stars' $E(V-R)_{R R}$ deviates by $0.3 \mathrm{mag}$ from $E(V-R)_{C M D}$, while the other one by only $0.06 \mathrm{mag}$. Other very blue stars include 129.26623.1139 with $(V-R)=0.24$ and 146.28412 .88 with $(V-R)=0.25$, but their $E(V-R)_{R R}$ is in agreement with $E(V-R)_{C M D}$. One more characteristic that 
distinguishes the blue outlying stars 175.30921 .95 and 125.23192 .175 from their blue nonoutlying counterparts, is the fact that these stars have periods that lie in the range 0.469-

0.472 days, a range in which unstable light curves are common (Kinman \& Carretta 1992). Spectra of unusually blue stars in the period interval of 0.469-0.472 days, AR Her and BB Vir, were taken by Sturch (1966) and Fernley \& Barnes (1996). It was found that these stars have systematically stronger hydrogen lines at minimum light than all the other stars, indicating a higher temperature. The cause of the higher temperature, however, remains unknown. Fernley \& Barnes (1996) suggested that BB Vir could be a RR0 Lyrae star that for some reason has evolved a long way beyond the fundamental blue edge without yet changing mode. The "hysteresis" effect is well-known in globular clusters and it is possible that stars 175.30921 .95 and 125.23192 .175 are also extreme examples of it. Stars at the blue edge of the instability strip should have a larger amplitude (Sandage 1981). Stars 175.30921.95 and 125.23192.175, the blue stars with deviating $E(V-R)$ values, have about twice as large $V$ amplitudes as the other two blue stars, 129.26623.1139 and 146.28412.88. It is also possible that the MACHO photometry of the star 175.30921 .95 and 125.23192 .175 is incorrect. Follow-up observations would help clarify this point. In conclusion, although rare, reddening values for RR0 Lyrae stars determined by finding the $(V-R)$ color at minimum $V$-band light can be in error if the star is unusually blue. This can be corrected if the temperature of the RR0 Lyrae star is known.

\section{Conclusions}

A quantitative analysis of reddening toward the Galactic Bulge is performed. Assuming that the extinction affects the $V$ magnitudes more significantly than the distance spread and the intrinsic scatter in absolute magnitudes, we interpret the slope of the stellar locus on the $V$ versus $(V-R)$ diagram as the selective extinction coefficient, $R_{V, V R}=A_{V} / E(V-R)$. From the MACHO RR0 Lyrae sample toward the Galactic Bulge, we derive $R_{V, V R}=4.3 \pm 0.2$. This corresponds to the average value observed in the solar neighborhood $R_{V, B V}=3.1 \pm 0.3$, and indicates that the optical reddening law toward the bulge is consistent with the standard extinction law. We show that the mean minimum-light $(V-R)_{0}$ color of an RR0 Lyrae star is nearly constant at minimum $V$-band light and equals $0.28 \pm 0.02$, regardless of metallicity. Using this property, reddening values to 3525 RR0 Lyrae stars are found. Parameters derived for individual stars are presented in the electronic version.

We would like to thank the referee, Derck Massa, whose thorough report has led to substantial improvements to this paper. Andrea Kunder thanks the Max-Planck-Institut für Astrophysik and Lawrence Livermore National Laboratory, where part of this work was 
completed, for their wonderful people and hospitality, especially Martin Jubelgas, David Syphers, and Andreas Hamm. KHC's and part of AMK's work was performed under the auspices of the U.S. Department of Energy by Lawrence Livermore National Laboratory in part under Contract W-7405-Eng-48 and in part under Contract DE-AC52-07NA27344. 


\section{REFERENCES}

Alcock, C., et al. 2000, ApJ, 542, 257

Alcock, C., et al. 1999, PASP, 111, 1539

Babu, J. \& Feigelson, E. D. 1992, Commun. Stat. Simulation 21, 533

Bessell, M.S. \& Germany, L.M. 1999, PASP, 111, 1421

Bessell, M.S. 1990, PASP, 91, 589

Blanco, V. 1992, AJ, 104, 734

Cacciari, C., Clementini, G., Prevot, L., Lindgren, H., Lolli, M., \& Oculi, L. 1987, A\&A, 69, 135

Cacciari, C., Clementini, G., Prevot, L., \& Buser, R. 1989a A\&A 209, 141

Cacciari, C., Clementini, G., \& Buser, R. 1989b A\&A 209, 154

Cacciari, C. \& Clementini, G., 2003, A\&A, 635, 105

Cardelli, J.A., Clayton, G.C. \& Mathis, J.S. 1989, ApJ, 345, 245

Clementini, G., Raffaele, G., Bragaglia, A., Carretta, E., Di Fabrizio, L, \& Maio, M. (2003) AJ, 125, 1309

Davidge, T. J. 1998, AJ, 115, 2374

Dutra, C. M., Santiago, B. X., Bica, E. L. D., \& Barbuy, B. 2003, MNRAS, 338, 253 (DSBB)

Eisenhauer, F., Schődel, R., Genzel, R., Ott, T., Tecza, M., Abuter, R., Eckart, A. \& Alexander, T. 2003, ApJ, 597, L121

Feigelson, E. D. \& Babu J. 1992, ApJ, 397, 55

Ferley, J. \& Barnes, T.G. 1996 A\&A 312, 957

Fitzpatrick, E.L. 1999, PASP, 111, 63.

Fogel, J.A., Tiede, G.P. \& Kuchinski, L.E. 1999 ApJ117, 2296

Gould, A. \& Popowski, P., 1998, ApJ, 508, 844

Hauschildt, P.H., Allard, F., Baron, E., 1999 ApJ, 512, 377 
Isobe, T., Feigelson, E. D., Akritas, M. G. \& Babu, G. J. 1990 ApJ364, 104

Kanbur, S. \& Fernando, I. 2004, preprint (astro-ph/0407485)

Kinman, T.D. \& Carretta, E. 1992 PASP 104, 111

Kunder, A. M., Popowski, P., Cook, K. H., Nikolaev, S. \& Chaboyer, B. 2006, ASPC 349, 395

Liu, T. \& Janes, K.A. 1989 ApJS, 69, 593

Mateo, M., Udalski, a. Szyma'nski, M., Kałużny, J., Kubiak, M. \& Krzemiński, W. 1995, AJ, 109, 588

Narayanan, V. K., Gould, A., \& DePoy, D. L. 1996, ApJ, 472, 183

Popowski, P. 2000, ApJ, 528, L9

Popowski, P., Cook, K.H., \& Becker, A. 2003, AJ, 126, 2910

Ruffle, P. M. E., Zijlstra A. A., Walsh J. R., Gray M. D., Gesicki K., Minniti D., \& Comeron F. 2004, MNRAS, 353, 796

Sandage, A. 1981, ApJ, 244, L23

Sandage, A. 1990, ApJ, 350, 603

Schlegel, D.J., Finkbeiner, D.P., \& Davis, M. 1998, ApJ, 500, 525

Stanek, K.Z. 1996, ApJ, 460, L37

Sturch, C. 1966, ApJ, 143, 774

Udalski, A. 2003, ApJ, 590, 284

Widrow L.M. \& Dubinski, J. 2005, ApJ631, 855 
Table 1: Slope $\left(R_{V, V R}\right)$ of 3256 Bulge RR0 stars plotted on a $V$ versus $(V-R)$ CMD.

\begin{tabular}{lcccc}
\hline Fit & Slope & Error & zero point & Error \\
\hline OLS bisector, all stars & 4.28 & 0.04 & 14.37 & 0.03 \\
OLS bisector, $(V-R)>0.34$ & 4.34 & 0.05 & 14.32 & 0.03 \\
OLS bisector, $(V-R)>0.34,(V-R)<1.11$ & 4.48 & 0.05 & 14.24 & 0.03 \\
\hline
\end{tabular}

Table 2: Derivation of minimum-light $(V-R)_{0}$ colors of field RR0 Lyrae stars

\begin{tabular}{lccccc}
\hline Star & $(V-R)$ & $E(V-R)$ & $(V-R)_{0}$ & {$[\mathrm{Fe} / \mathrm{H}]$} & $V$ Amp \\
\hline SWAnd & 0.34 & 0.035 & 0.295 & -0.24 & 0.89 \\
RRCet & 0.30 & 0.019 & 0.301 & -1.45 & 0.78 \\
RXEri & 0.34 & 0.053 & 0.277 & -1.33 & 0.87 \\
RRGem & 0.35 & 0.054 & 0.276 & -0.29 & 1.15 \\
RRLeo & 0.31 & 0.034 & 0.266 & -1.60 & 1.23 \\
TTLyn & 0.30 & 0.014 & 0.286 & -1.56 & 0.70 \\
AVPeg & 0.34 & 0.056 & 0.274 & -0.08 & 1.08 \\
TUUMa & 0.30 & 0.018 & 0.272 & -1.51 & 1.02 \\
SSFor & 0.27 & 0.012 & 0.248 & -0.94 & 1.23 \\
RVPhe & 0.30 & 0.006 & 0.284 & -1.69 & 0.71 \\
V440Sgr & 0.33 & 0.076 & 0.254 & -1.40 & 1.16 \\
\hline
\end{tabular}

Table 3: Fragment of the catalog of reddenings for 3525 RR0 Lyrae stars in the MACHO Galactic bulge fields

\begin{tabular}{lcccccccccc}
\hline Star ID & Ra & Dec & Gal $l$ & Gal $b$ & Period & $V$ mag & $R$ mag & $V$ amp & $(V-R)_{\min } E(V-R)$ \\
\hline 101.20648 .645 & $18: 04: 31.80$ & $-27: 33: 55.8$ & 3.286 & -2.904 & 0.590062 & 17.58 & 16.77 & 0.57 & 0.808 \\
101.20649 .500 & $18: 04: 21.36$ & $-27: 27: 44.6$ & 3.357 & -2.820 & 0.570140 & 17.11 & 16.46 & 0.70 & 0.729 \\
101.20650 .531 & $18: 04: 25.32$ & $-27: 26: 16.1$ & 3.385 & -2.821 & 0.557547 & 17.29 & 16.44 & 0.81 & 0.723 \\
101.20650 .965 & $18: 04: 24.24$ & $-27: 27: 40.7$ & 3.363 & -2.829 & 0.505202 & 18.38 & 17.57 & 0.94 & 0.829 \\
101.20652 .684 & $18: 04: 23.16$ & $-27: 17: 18.2$ & 3.512 & -2.740 & 0.570760 & 17.07 & 16.36 & 0.25 & 0.720 & 0.45 \\
101.20653 .526 & $18: 04: 25.32$ & $-27: 11: 42.4$ & 3.597 & -2.702 & 0.469922 & 17.26 & 16.53 & 0.76 & 0.688 & 0.44 \\
101.20654 .508 & $18: 04: 30.72$ & $-27: 11: 14.6$ & 3.614 & -2.716 & 0.552941 & 17.13 & 16.33 & 0.88 & 0.824 \\
101.20654 .938 & $18: 04: 32.88$ & $-27: 08: 09.6$ & 3.663 & -2.697 & 0.457945 & 17.51 & 16.78 & 0.70 & 0.778 & 0.54 \\
101.20778 .1172 & $18: 04: 36.84$ & $-27: 35: 11.4$ & 3.276 & -2.930 & 0.514020 & 17.76 & 16.95 & 0.99 & 0.817 & 0.50 \\
101.20779 .378 & $18: 04: 41.16$ & $-27: 28: 17.4$ & 3.385 & -2.888 & 0.633424 & 17.06 & 16.26 & 0.53 & 0.835 & 0.55 \\
\hline
\end{tabular}




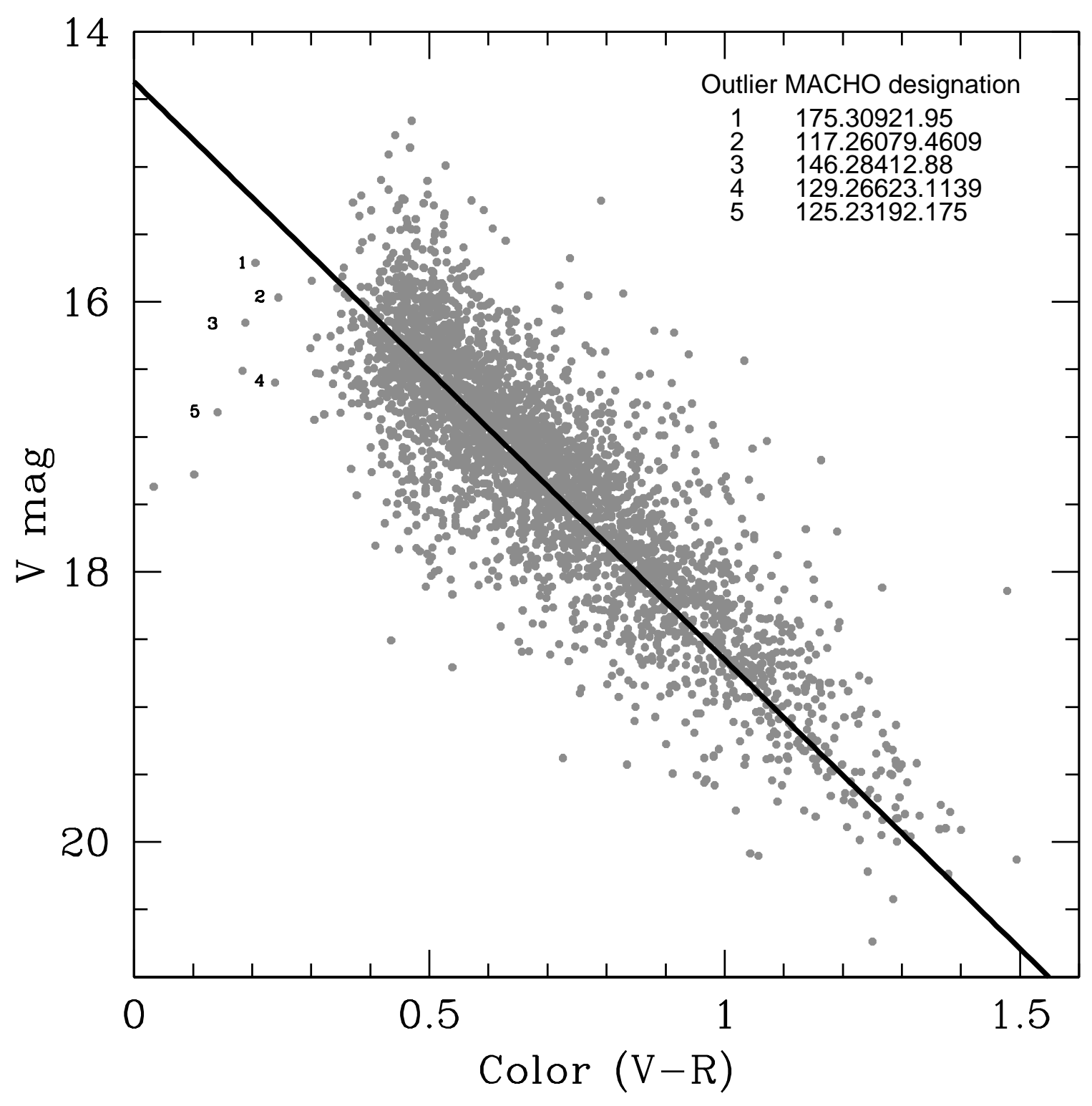

Fig. 1.- Color-magnitude diagram for the 3256 MACHO Galactic Bulge RR0 Lyrae stars. The best fit mean $V$ versus mean $(V-R)$ relation is shown by the solid line: $V_{R R}=$ $(4.28 \pm 0.04)(V-R)_{R R}+(14.37 \pm 0.03)$. and was obtained using the OLS-bisector method (Feigelson \& Babu 1992), . 


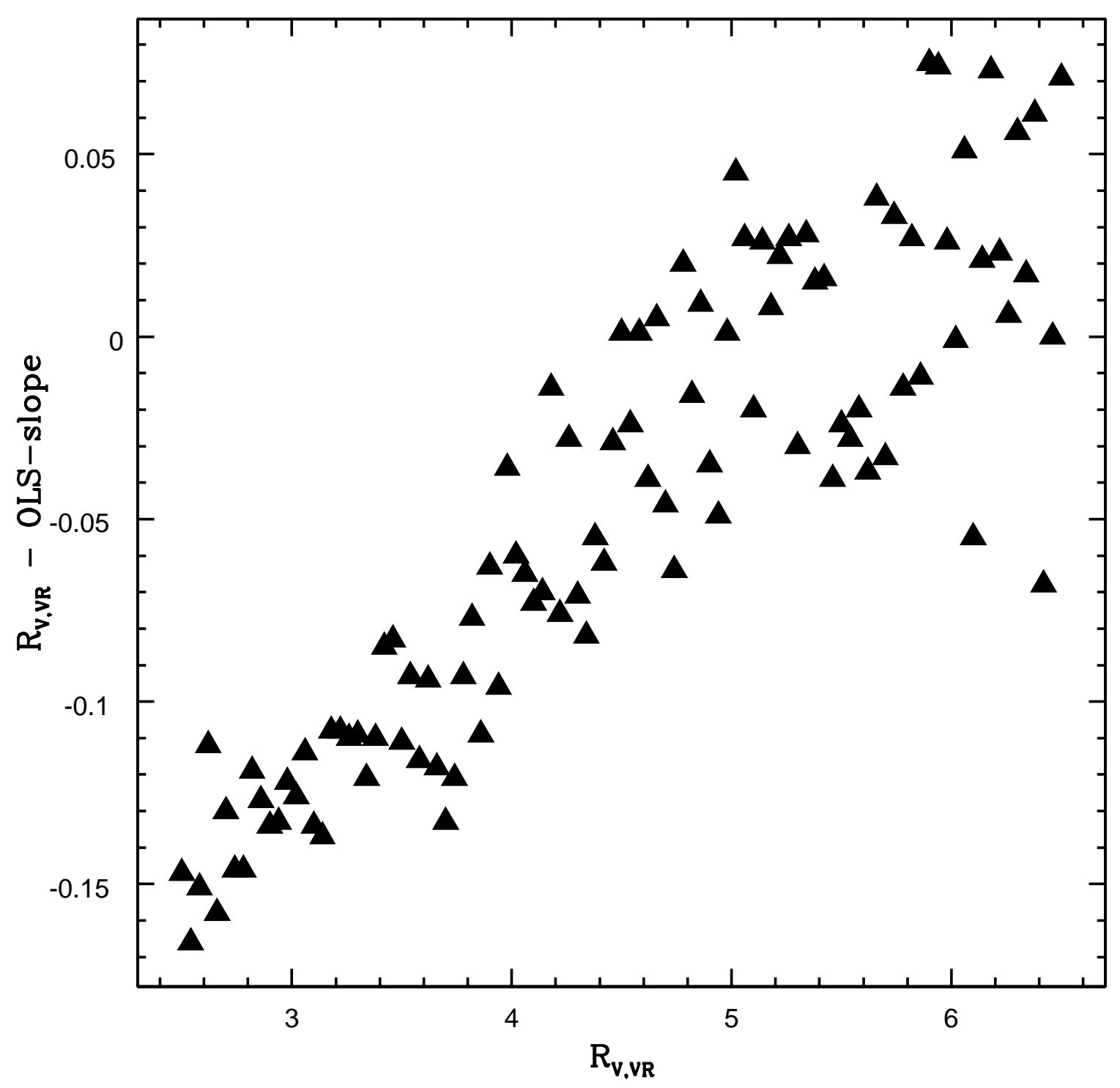

Fig. 2.- The difference in the true $R_{V, V R}$ value and the OLS-bisector slope, $R_{(V, V R)}$ OLS-fitted slope, as a function of the true $R_{V, V R}$ value. 


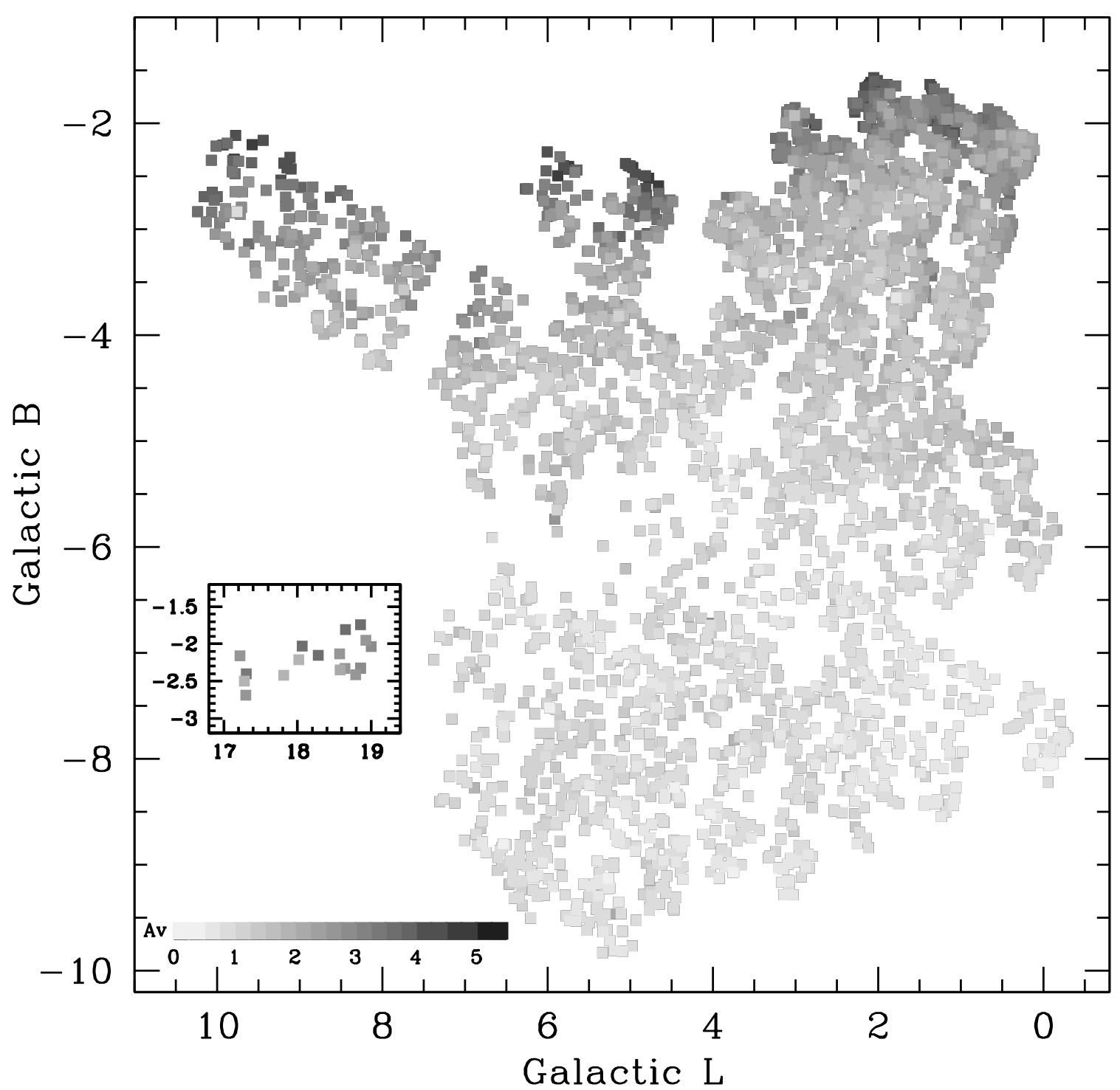

Fig. 3.- Reddening map of $A_{V}$ values of the central Galactic region. The scale is given at the bottom. Insert presents three disk fields separated from the others by $10^{\circ}$. 


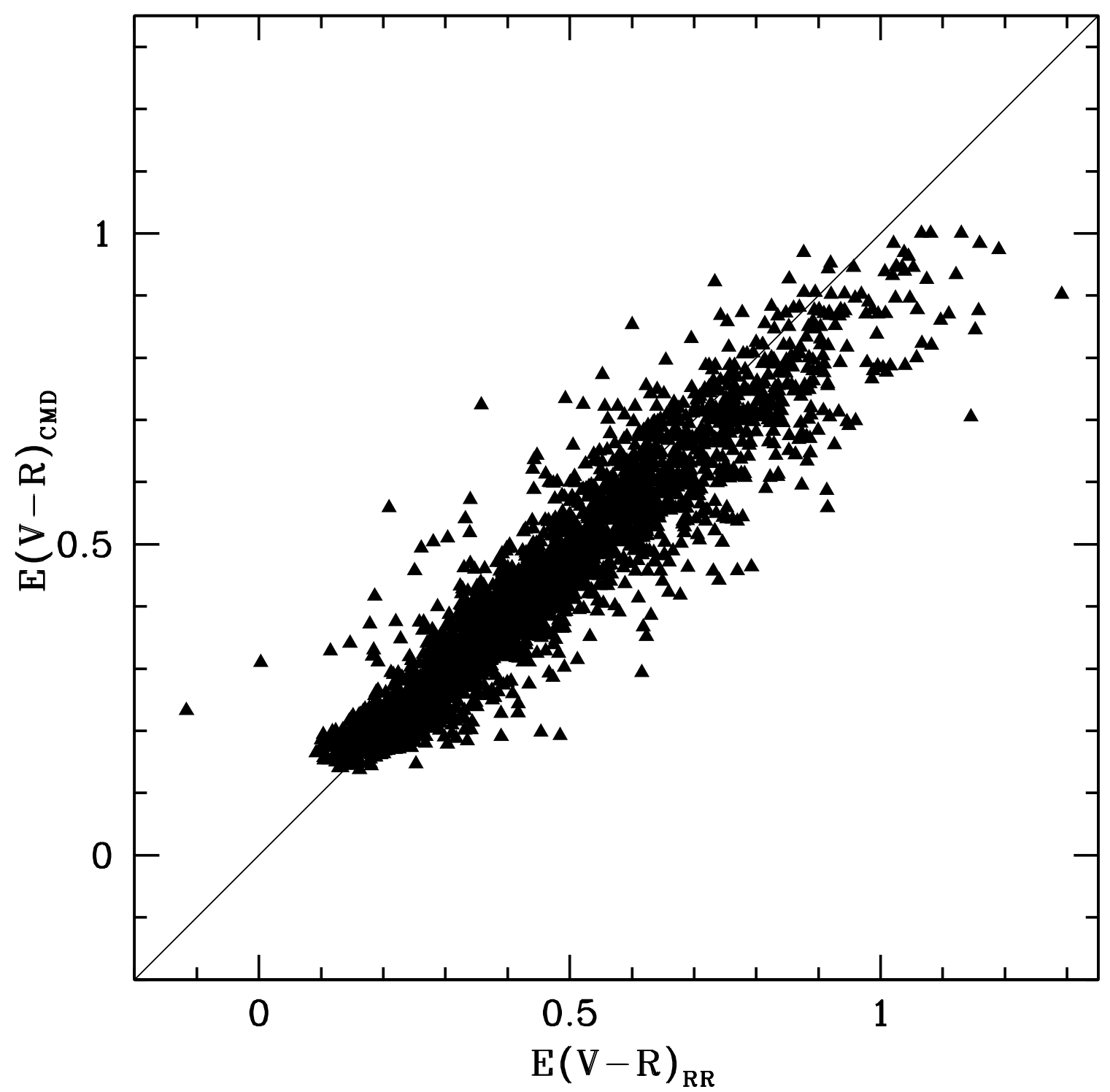

Fig. 4.- A comparison of $E(V-R)$ derived using $(V-R)$ at minimum light, $E(V-R)_{\mathrm{RR}}$, for $2605 \mathrm{RR}$ Lyrae stars with color-magnitude based $E(V-R)_{\mathrm{CMD}}$ from Popowski et al. (2003). The relation is linear, with $E(V-R)_{\mathrm{RR}}$ values slightly larger than $E(V-R)_{\mathrm{CMD}}$ at high extinction. 